\title{
Continuous Simulation of Catchment Runoff in Flood Frequency Analysis: A Case Study from Slovakia ${ }^{\dagger}$
}

\author{
Peter Valent * and Roman Výleta \\ Department of Land and Water Resources Management, Faculty of Civil Engineering, Slovak University of \\ Technology in Bratislava, 81005 Bratislava, Slovakia; roman.vyleta@stuba.sk \\ * Correspondence: peter_valent@stuba.sk; Tel.: +421-2-59-274-727 \\ + Presented at the 3rd International Electronic Conference on Water Sciences, 15-30 November 2018; \\ Available online: https://ecws-3.sciforum.net.
}

Published: 15 November 2018

\begin{abstract}
Research questions relating to a reliable estimate of flood discharge have always interested both hydrologists and civil engineers. Over the decades, numerous methods have been proposed and used more or less successfully, all of them with known limitations restricting their use in a wide range of conditions and problems. In the past, the characteristics of hydrological extremes were mostly estimated by the methods of statistical analyses. As this type of method is not suitable to estimate design discharges of high return periods, and by default does not account for uncertainty, a new family of methods is slowly taking the place of the traditional approaches. Many of these methods are based on a combination of stochastic rainfall models (weather generators) and rainfallrunoff models, which enables generation of an arbitrary number of synthetic floods, even in places with short or no record of river discharges available. In addition, as this type of method produces flood hydrographs, they can also be used in a multivariate flood frequency analysis to estimate joint probabilities of two or more flood characteristics. This study presents a methodology for flood frequency analysis that combines stochastic models of both rainfall amounts and air temperatures with a lumped rainfall-runoff model to transfer the outputs of the stochastic models into a series of corresponding river discharges. Both of the stochastic models are single-site weather generators that produce continuous time series of mean areal daily rainfall amounts and air temperatures. In this study, the method was used to generate a time series of 10,000 years of mean daily discharges, which was used to build a flood frequency curve and to estimate extreme flood discharges of given return periods. The method was applied to a mountainous catchment of the River Váh in Slovakia.
\end{abstract}

Keywords: flood frequency analysis; rainfall-runoff model; weather generator; stochastic rainfall model

\section{Introduction}

Most of the methods dealing with flood frequency analyses (FFA) and the estimation of design floods are based on statistical analysis of the observed time series of flows. This traditional concept proved to be reliable when estimating design floods with return periods of less than 100 years and in places with long historical records. Nowadays, there is a trend in water management for increasing the safety of new and existing structures, such as dams, dykes, and other flood mitigation structures (e.g., in France and the Czech Republic, all new and existing dams must be (re)designed to withstand a 10,000-year flood). There is also a slow movement from univariate to multivariate statistical analysis, which studies the joint probabilities of more than one flood characteristic (e.g., a flood peak, volume, and duration) [1-3]. Moreover, the evaluation of uncertainties of design flood estimates, 
together with providing their confidence bounds, has become a standard in many countries. All these new tasks reveal well-documented weaknesses of the traditional methods, among which the insufficient length of historical records is the most importantone.

One of the possible ways to deal with these new challenges is to artificially lengthen the historical records by synthetic data. In recent years, science worldwide has focused on the development of methods utilizing stochastic weather generators to generate synthetic meteorological and climatic data of arbitrary lengths and rainfall-runoff (r-r) models to transform them into synthetic runoff from a catchment. Such models are still under development [4-6], and most hydrologists and practitioners still use the traditional methods for statistical analyses of observed flows.

This study presents a method for a continuous flood frequency analysis, which utilizes both a weather generator and an r-r model. The method was applied in the Slovak catchment of the River Váh with an outlet at Liptovský Mikuláš station. The study is mainly focused on an assessment of the simulation of both extreme precipitation and flows.

\section{Materials and Methods}

The method for the continuous flood frequency analysis presented in this study was developed at the Slovak University of Technology in Bratislava [7,8]. The method utilizes a WGbRV stochastic weather generator (WG) [7] to generate 10,000 years of daily precipitation amounts and air temperatures and a KZOM r-r model [8], which is used for their transformation into a time series of synthetic flows. The time series of flows is used to build a flood frequency curve from an annual maxima (AM) series of flows. The daily discharges of a given return period that were estimated from the flood frequency curve are then transformed into peak discharges using a simple peak-to-volume ration [9]. Its value is obtained by analyzing a large number of hourly flood hydrographs.

\subsection{Weather Generator and Rainfall-Runoff Models}

The weather generator used is a single site WG. The synthetic time series of precipitations is generated in the following three steps. In the first step, a model of precipitation of occurrence is used to generate a time series of the wet and dry days. In the second step, precipitation amounts are generated for each rainfall event using a statistical distribution. In the third step, the precipitation amounts are disaggregated into the individual days of rainfall events using a method of fragments $[7,10]$. The corresponding time series of air temperatures is generated separately. The model takes into account both the current calendar month and the information about the occurrence of precipitation on a given day. The model utilizes harmonic functions and an autoregressive model of the first order.

The KZOM r-r model used in the method is a conceptual deterministic model based on the Swedish HBV model [11]. The model works in a daily time step with a lumped representation of the parameter space. Apart from time series of precipitation amounts, air temperatures, and flows for model calibration, the model also requires estimates of mean daily potential evapotranspiration. In order to improve the simulation of extreme flows, a two-regime scheme of the model was used. It uses different routing parameters to simulate (a) low and medium flows, and (b) high flows. The decision as to which regime is used depends on the value of the flow from the previous day. The value of the threshold that determines whether the flow falls into regime one or two is optimized using a genetic algorithm. The two sets of model parameters were obtained in a two-step model calibration utilizing both Nash-Sutcliffe efficiency criterion (low and medium flows) and an aggregate optimization function (high flows) [9].

\subsection{Data}

The method for flood frequency analysis that is described above was applied in the mountainous catchment of the River Váh with an outlet at the Liptovský Mikuláš water gauge (1-4-21-02-027-01). The catchment is situated in the northern part of Slovakia between the two highest Slovak mountain ranges, i.e., the High Tatras in the north and the Low Tatras in the south (see Figure 1). It has an area 
of $1100.6 \mathrm{~km}^{2}$ with a mean altitude of 1090.3 m.a.s.l. and the highest point at 2494 m.a.s.l. The water regime has a strong seasonal effect, with the highest flows in May and the lowest in the winter months. Most of the floods occur in May and are induced by the rapid melting of snow cover combined with steady rain. The mean annual precipitation amount totals for $832 \mathrm{~mm}$. From this amount, $553 \mathrm{~mm}$ of precipitation ends up in the catchment's outlet, which corresponds to a mean annual river discharge of $19.3 \mathrm{~m}^{3} / \mathrm{s}$. The daily flow data, which were available for the analysis, spanned a period between 1981 and 2010. The daily precipitations (28 rain gauge stations used) and the daily air temperatures (6 climatic stations used) were used to calculate time series of areal precipitations and air temperatures for a period between 1981 and 2010. As the catchment selected lies in the upper part of the whole watershed of the River Váh and it is not a subject of intensive anthropogenic activities, as much as $50 \%$ of its area is covered by forest. Arable land constitutes only $16 \%$ or the totalarea.
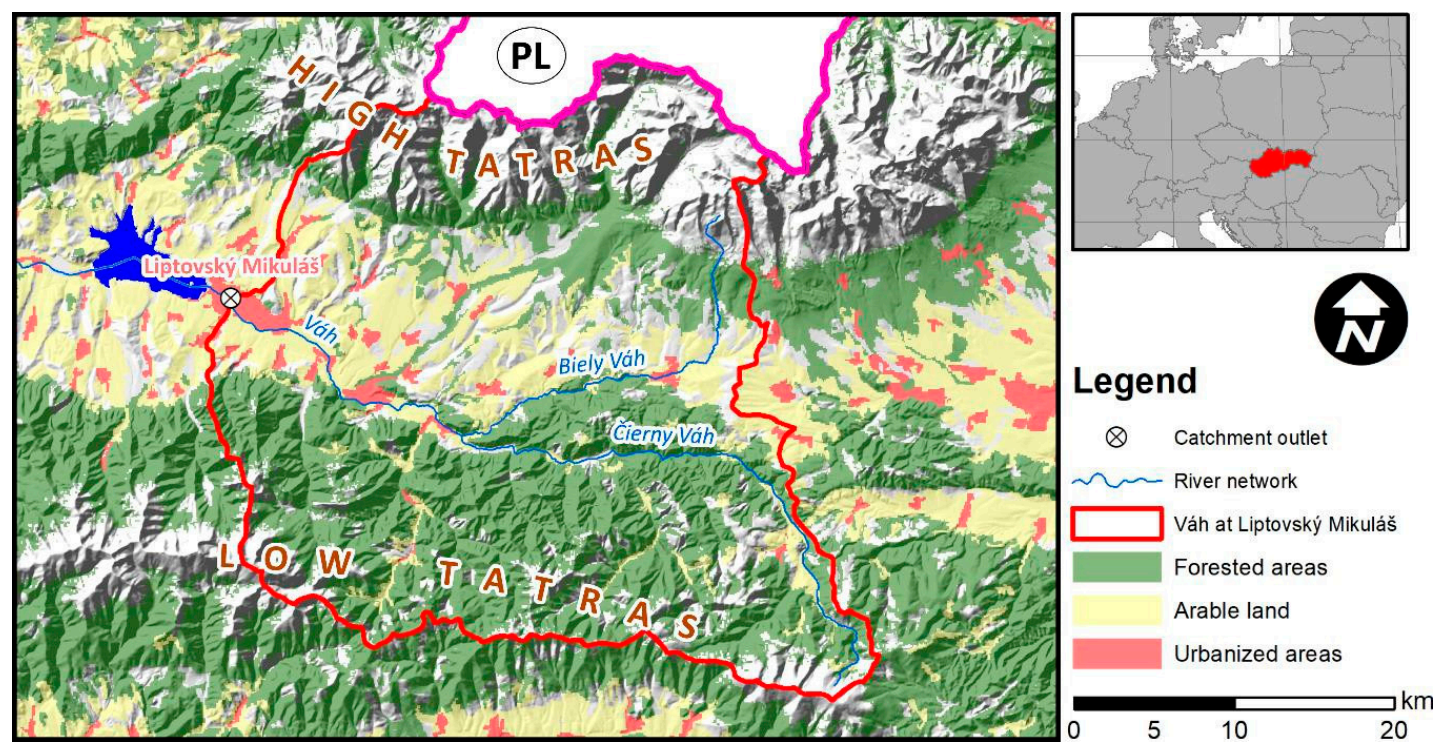

Figure 1. Position of the watershed of the River Váh in Slovakia.

\section{Results}

\subsection{Generating Synthetic Inputs}

The weather generator described in the methodology was used to generate synthetic time series of the mean areal daily rainfall totals and air temperatures. The length of the generated time series was 10,000 years, which should ensure that the synthetic time series would contain precipitation events of such intensities that were not observed in the historical records. Moreover, the length of such time series should comprise rare combinations of extreme rainfall events and adverse initial conditions, such as extreme rainfall on a saturated watershed. Since the generation of synthetic air temperatures requires information about the occurrences of precipitation, the time series of synthetic precipitation was generated first.

The model of precipitation occurrence was used to generate a time series of wet and dry days. It accounted for seasonality and used a Markov model to decipher whether a given day is wet or dry. The order of the Markov model was optimized for each month and was 1 for January to September and 2 for October to November. The precipitation amounts were generated by appropriate statistical distributions fitted for each combination of m-day precipitation and month. The quality of the generated precipitation time series was assessed by comparing its ability to reproduce the statistical characteristics of the historical records in both daily and annual maxima series domains. The results showed that the model was able to reproduce the distribution of the length of both dry and wet periods very well (see Figure 2a for wet periods). Moreover, the model also performed well in reproducing the mean $m$-day precipitation amounts (Figure2b). 


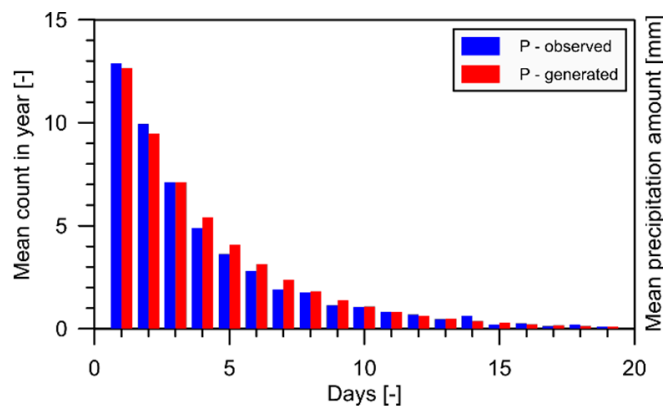

(a)

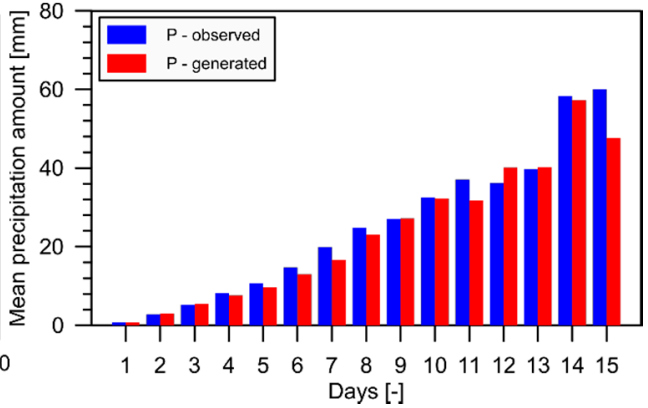

(b)

Figure 2. Comparison of selected statistical characteristics of observed and generated precipitation time series: (a) The mean number of wet periods of a particular length in a year; (b) the mean m-day precipitation amount of a particular duration.

As the FFA focuses on extreme flows, a good simulation of extreme precipitation is essential. In order to assess the ability of the statistical rainfall model to reproduce the statistical characteristics of extreme precipitations, AM series of both observed and generated precipitations were created and compared as histograms and empirical cumulative distribution functions (ECDF) (Figure 3a,b). The results show that the generated AM precipitation series contains less values in a range between 35$40 \mathrm{~mm}$ and $45-50 \mathrm{~mm}$ than the corresponding observed series. Nevertheless, the differences between the two histograms (Figure 3a) are natural due to the short length of the observed AM series. The model also generates a certain amount of precipitation, which is higher than the maximum precipitation in the historical records (see Figure $3 a, b$ ). Since the statistical distributions used to generate the precipitation amounts occasionally enable the generation of unrealistic values, a threshold determining the maximum possible daily precipitation was set as a double of the mean values of the 10 highest precipitation amounts observed in the historical records [8]. The fact that the model generates a certain amount of precipitation higher than the maximum precipitation in the historical records while preserving its selected statistical characteristics makes it suitable for use in flood frequency analysis.

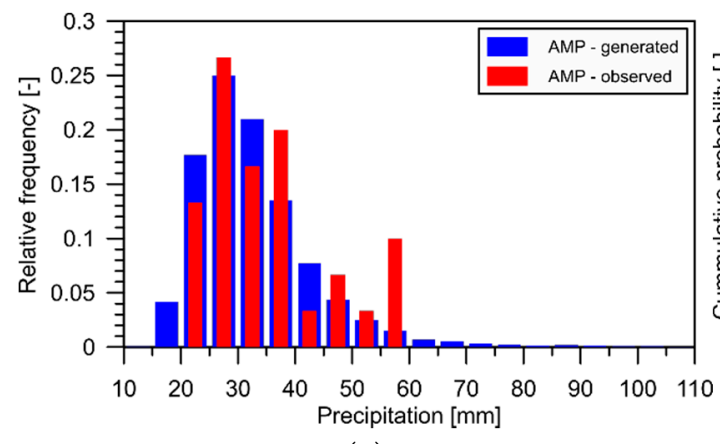

(a)

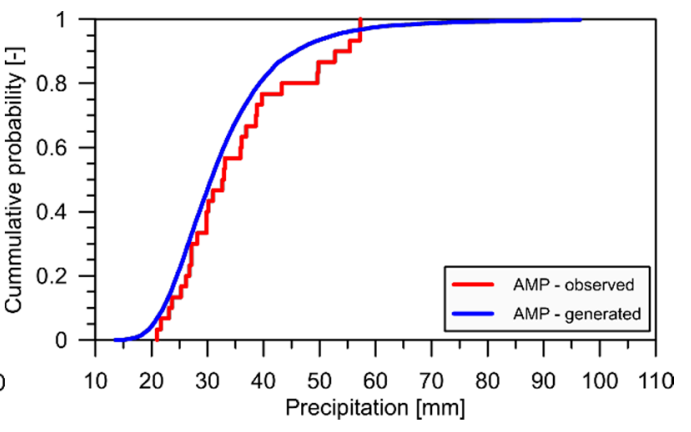

(b)

Figure 3. Comparison of the annual maxima series of observed and generated precipitations in the form of: (a) Histogram; (b) empirical cumulative distribution function.

\subsection{Generating Synthetic Flows}

The KZOM r-r model was calibrated using the historical records of flows (the period between 1981 and 2010) to obtain two sets of parameters for the simulation of low and medium flows, and high flows. The value of the Nash-Sutcliffe efficiency criterion (NS) calculated for the whole period was 0.70 . By comparing the interannual regime of the observed and simulated flows (Figure 4a) one might notice that the highest errors occur during the summer period. On the other side, the simulation of the extreme flows is rather good with no significant differences between the observed and simulated ECDF (Figure $4 \mathrm{~b}$ ). In the next step, the model was used to transform the synthetic inputs into a synthetic (generated) time series of flows. The transformation produced a synthetic time 
series of mean daily flows with a length of 10,000 years. The position of the ECDF estimated for the synthetic flows indicates that the model slightly underestimates extreme flows when compared to the ECDFs of the observed and simulated flows (Figure $4 \mathrm{~b}$ ). Nevertheless, the model was able to generate a certain number of floods higher than those presented in the historical records. This resulted in the AM series of synthetic flows containing 38 peak flows that were higher than the largest one observed.

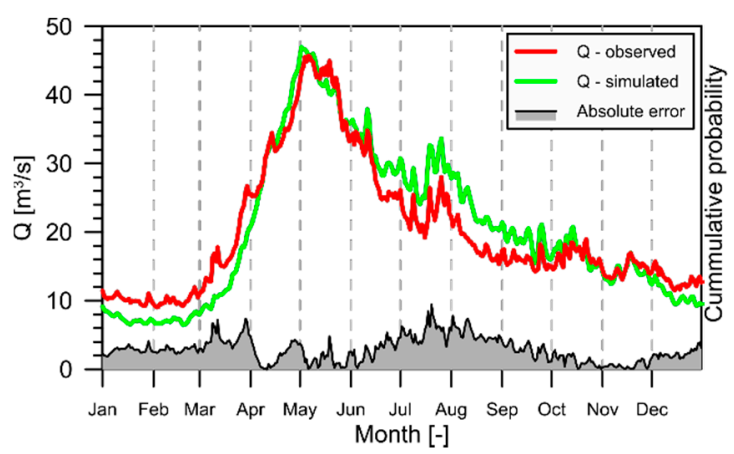

(a)

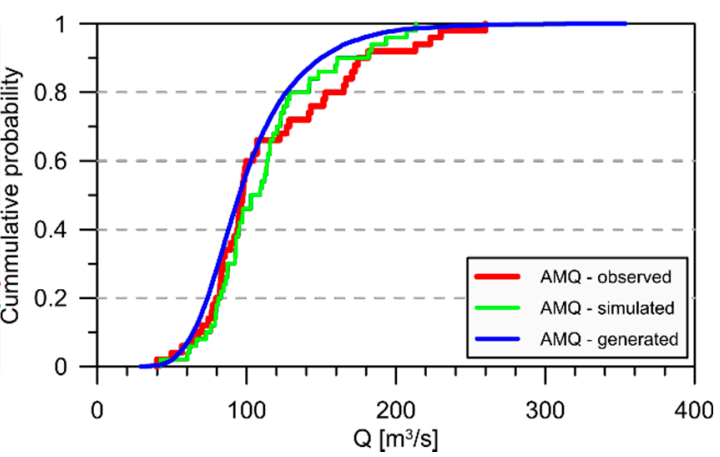

(b)

Figure 4. Comparison of: (a) Interannual flow regimes of observed and simulated flows; (b) ECDFs of the AM series of the observed, simulated, and synthetic (generated) flows.

\section{Discussion}

The method for flood frequency estimation presented here follows the trend of the last decades and builds on the advantages of weather generators and continuous r-r simulations. If working properly, it could be used to reliably estimate peak flows of very high return periods, and thus meet the new requirements to reassess the risks associated with existing engineering structures (e.g., dams, flood protection of large cities).

Moreover, the continuous and uninterrupted nature of the synthetic flows would allow for the use of a multivariate flood frequency analysis, which would enable association of probabilities to, for example, both flood peaks and volumes. Furthermore, as WGs allow for the generation of synthetic inputs that are relatable to whole watersheds it is only a question of a correct calibration of an r-r model to generate the synthetic flows in ungauged watersheds. Despite the fact that the WG used replicated the basic statistical characteristics of the historical records, an accurate and reliable simulation of rainfall extremes is still a challenge. Figure 3a shows that the generated AM series of precipitations comprised much fewer values (in terms of frequency) in a range between 55 and $60 \mathrm{~mm}$ than the one observed. Nevertheless, a much bigger challenge is associated with large watersheds, in which case the generated inputs should be spatially distributed to account for the large spatial variability of precipitation in such watersheds.

However, in this study, the biggest problems were related to the r-r modelling. The great steepness of the hypsometric curve called for a semi-distributed modelling approach, in which the model inputs would be divided into several elevation zones. As the WG used is only a single-site generator, this was not possible. Even though the overall performance of the model was good, it was not able to correctly simulate the snow melting and accumulation processes, and thus the most extreme floods that occur in the late spring.

\section{Conclusions}

The study presented a simple methodology for flood frequency estimation utilizing a single-site weather generator to generate 10,000 years of mean areal daily precipitations and air temperatures and a conceptual $r-r$ model that transforms them into a time series of flows. The method was applied to a Slovak mountainous catchment of the River Váh. The results exposed some of the limitations of the method presented that were mostly related to problematic simulations of both extreme precipitations and flows. 
Therefore, further research should focus on the development of a multi-site WG that would account for a large spatial variability of precipitations, better calibration procedures of r-r models to correctly simulate extreme flows, and the transferability of model parameters to ungauged basins.

Author Contributions: P.V. was responsible for data preparation, developing the rainfall-runoff model and its calibration, and writing the paper. R.V. developed the weather generator and generated the synthetic input time series.

Funding: This research was funded by the Slovak Research and Development Agency grant number APVV-150497 and the VEGA grant agency grant number VEGA 1/0891/17. The APC was funded by the VEGA grant agency grant number VEGA 1/0891/17.

Conflicts of Interest: The authors declare no conflict of interest.

\section{References}

1. Papaioannou, G.; Kohnová, S.; Bacigál, T.; Szolgay, J.; Hlavčová, K.; Loukas, A. Joint modelling of flood peaks and volumes: A copula application for the Danube River. J. Hydrol. Hydromech. 2016, 64, 382-392, doi:10.1515/johh-2016-0049.

2. Szolgay, J.; Gaál, L.; Bacigál, T.; Kohnová, S.; Hlavčová, K.; Výleta, R.; Parajka, J.; Blöschl, G. A regional comparative analysis of empirical and theoretical flood peak-volume relationships. J. Hydrol. Hydromech. 2016, 64, 367-381, doi:10.1515/johh-2016-0042.

3. Gaál, L.; Szolgay, J.; Bacigál, T.; Kohnová, S.; Hlavčová, K.; Výleta, R.; Parajka, J.; Blöschl, G. Similarity of empirical copulas of flood peak-volume relationships: A regional case study of North-West Austria. Contrib. Geophys. Geod. 2016, 46, 155-178, doi:10.1515/congeo-2016-0011.

4. Madsen, H.; Lawrence, D.; Lang, M.; Martinkova, M.; Kjeldsen, T.R. (eds.) A Review of Applied Methods in Europe for Flood-Frequency Analysis in a Changing Environment; Centre for Ecology \& Hydrology: Wallingford, UK, 2013; ISBN 978-1-906698-36-2.

5. Blazkova, S.; Beven, K. Flood frequency estimation by continuous simulation of subcatchment rainfalls and discharges with the aim of improving dam safety assessment in a large basin in the Czech Republic. J. Hydrol. 2004, 292, 153-172, doi:10.1016/j.jhydrol.2003.12.025.

6. Boughton, W.; Droop, O. Continuous simulation for design flood estimation-A review. Environ. Model. Softw. 2003, 18, 309-318, doi:10.1016/S1364-8152(03)00004-5.

7. Výleta, R. Modelling Areal Precipitation Amounts for Flood Frequency Analysis. Ph.D. Thesis, Slovak University of Technology in Bratislava, Bratislava, Slovakia, 2013.

8. Valent, P. Rainfall-Runoff Modelling for Flood Frequency Analysis. Ph.D. Thesis, Slovak University of Technology in Bratislava, Bratislava, Slovakia, 2014.

9. Paquet, E.; Garavaglia, F.; Garçon, R.; Gailhard, J. The SCHADEX method: A semi-continuous rainfallrunoff simulation for extreme flood estimation. J. Hydrol. 2013, 495, 23-37, doi:10.1016/j.jhydrol.2013.04.045.

10. Svanidze, G.G. Mathematical Modeling of Hydrologic Series for Hydroelectric and Water Resources Computations; Water Resources Publications: Forth Collins, CO, USA, 1980; ISBN 978-091-833-432-9.

11. Bergström, S. Principles and Confidence in Hydrological Modelling. Hydrol. Res. 1991, 22, 123-136, doi:10.2166/nh.1991.0009.

(C) 2018 by the authors. Licensee MDPI, Basel, Switzerland. This article is an open access article distributed under the terms and conditions of the Creative Commons Attribution (CC BY) license (http://creativecommons.org/licenses/by/4.0/). 